\title{
Liberaler Kosmopolitismus: Eine partikularistische Emanation mit Unverträglichkeiten und Nebenwirkungen
}

\section{Das kosmopolitische Projekt}

Unter Kosmopolitismus (Held 2003) verstehe ich eine Vorstellung, die prinzipiell (mit naturrechtlicher Begründung) oder historisch (unter Hinweis auf die durch die Globalisierung entstandene Vernetzung der Menschheit) eine globale Vergemeinschaftung unterstellt, die als Grundlage für ein minimales, durchsetzungsfähiges Weltbürgerrecht (»dünne Version«) oder gar für ausdifferenzierte, weltrechtsgestützte Formen verbindlichen und sanktionsfähigen Regierens mit allgemeiner Teilhabe (»dichte Version«) tauge. Beide Versionen ruhen auf der Vorstellung, dass sich universalistische Prinzipien widerspruchsfrei und unabhängig von partikularistischen Ethiken a priori deduzieren ließen und daher aus sich heraus Geltung für alle beanspruchen könnten. Der kosmopolitische Diskurs vertritt die - gelegentlich auch explizit geäußerte (z. B. Beitz 1979; Pogge 2002; Kuper 2004; Höffe 2002) - Doktrin nicht nur der Zustimmungsfähigkeit, sondern gar der Zustimmungspflichtigkeit der von ihm propagierten Normen.

Kennzeichnend für das kosmopolitische Projekt ist der doppelte Anspruch, sowohl eine empirisch gültige, angemessene Beschreibung des politischen Weltzustands vorzulegen als auch moralisch gültige, praxeologische Forderungen daran zu knüpfen. Im Allgemeinen geschieht dies mit eindrucksvollem Selbstbewusstsein und beachtlicher Vehemenz. Beide Ansprüche halte ich für nicht vertretbar, weil sie nur durch das beharrliche Ignorieren des partikularistischen, kulturgebundenen Standpunkts zustande kommen können, welcher trotz seiner universalistischen Emphase dem kosmopolitischen Projekt anhaftet.

\section{Globalisierung und Fragmentierung}

Die Globalisierung bringt in der Tat beachtliche Konvergenzen, Amagalmierungen, Vernetzungen und Kommunikationen zwischen zuvor weitgehend getrennten kulturellen Räumen mit sich (Beisheim et al. 1999). Sie weicht die harte Schale des Nationalstaats vergleichsweise wirkungsvoll auf, obgleich der Idealtypus eines völlig nach außen abgegrenzten Kokons nie der Realität des westfälischen Systems entsprochen hat oder entsprechen konnte (Krasner 1999). Für die Verwirklichung gesellschaftlicher Ziele und Projekte ist immer die Mitwirkung, das Tun und Lassen, von Akteuren außerhalb der Grenzen des Territorialstaates vonnöten - seien es andere Staaten oder nichtstaatliche Akteure. Wieder ist jedoch darauf zu verweisen, 
dass absolute Autarkie zwar hin und wieder die Ideologie, in der Praxis indes nie die Realität nationalstaatlicher Daseinsvorsorge gewesen ist. Entsprechende Programme in der Nachfolge des Merkantilismus waren hochgradig pathologisch und infolgedessen auch kriegstreibend und im Sinne des effizienten Erreichens nationaler Ziele ausgesprochen suboptimal. Jedoch haben die Vernetzungen zugegebenermaßen heute eine neue Qualität erreicht.

Diese Entwicklung ist jedoch nur eine Seite der Medaille. Die andere Seite ist die Selbstversicherung partikularer Güter und Werte, namentlich durch solche Akteure, die unter der gewaltsamen Ausdehnung der westlichen Welt für Jahrhunderte jeder Chance zur Autonomie beraubt waren (Müller 1998: Kap. II.1). Aber auch in der westlichen Welt sind die Reaktionen der Selbstbehauptung stark, wie die vielbeachtete »Renationalisierung « europäischer Politik, der unverkennbare Nationalismus der Vereinigten Staaten oder die Europa-Aversionen der Schweizer und der Norweger dokumentieren (Rupnik 2006). Mit anderen Worten, die Globalisierung hat als ihren siamesischen Zwilling die Fragmentierung bei sich (Clark 1997; Zürn 2001), und das hat für das Kosmopolitismus-Projekt auf absehbare Zeit hinderliche Folgen. Zurück zu den »Underdogs « der vergangenen drei Jahrhunderte. Staatliche Souveränität wird dort vielfach - und zwar auch von Menschen, die unter den unzulänglichen bis tyrannischen Formen der Herrschaft leiden und dagegen kämpfen - als Schutz der Chance einer autonomen Entwicklung verstanden. Dass etwa viele oppositionelle Iraner den gewaltsamen Regimewechsel zurückweisen, obwohl sie das gegenwärtige System als abscheulich empfinden, ist indikativ: Die Herrschenden sind zwar ungeliebte Antidemokraten, aber es sind für sie »unsere « Antidemokraten. Zwar ist diese Einstellung nicht allgemein; aber die Präferenzordnung, die Autonomie vor Demokratie setzt, ist verbreitet genug, um über die kosmopolitische Emphase noch einmal nachzudenken.

\section{Liberaler Kosmopolitismus als kulturell gebundener Partikularismus}

Was selbst die kosmopolitischen Minimalisten als universal gültig vortragen, ist ideengeschichtlich samt und sonders ein Produkt der westlichen liberalen Geschichte und Kultur, deren Modellcharakter für den Rest der Welt man unterstellt (Hopgood 2000). Es ist lehrreich, einmal ganz bewusst die Balken im eigenen Auge zu besichtigen: Wir haben ein System erfunden, das in zunehmendem Maße begüterten und organisierten Interessen einen disproportionalen Einfluss auf politische Entscheidungen gestattet, Meinungsmache in immer weniger Händen konzentriert, soziale Ungleichheiten (und damit massive Chancenungleichheiten) aufrechterhält und jüngst wieder vergrößert. Unsere Vorstellung von Freiheit gestattet die ständige Verletzung der Gefühle des religiösen Teils der Bevölkerung und die Abwertung des weiblichen Körpers als Schaustück des männlichen Voyeurismus und als kommerzielles Werbeobjekt. Ungeachtet verfassungsmäßiger Bestimmungen und liberaler Rhetorik ist die Diskriminierung (bis zur Drangsalierung) des Fremden fester Teil unserer gesellschaftlichen und politischen Praxis. Unser System pflegt einen fast 
blasphemischen Umgang mit der Schöpfung und entbehrt eines belastbaren Konzepts für die ökologische und soziale Zukunftsvorsorge. Es hat die Großfamilie zerlegt und schickt sich an, dasselbe mit der Kernfamilie zu tun. Die Vernachlässigung der Kinder und die Entwürdigung der Alten scheinen seine zwangsläufigen Folgen zu sein. Der Drogenkonsum ist verbreitet und wird nicht wirksam bekämpft.

Ich selbst fühle mich in der spezifisch liberal geprägten Umwelt, deren Schattenseiten ich gerade versucht habe zu benennen, ausgesprochen wohl. Das liegt daran, dass ich darin groß geworden und folgerichtig enkulturiert bin. Den Angehörigen anderer Kulturen und Regionen muss man es aber wohl nachsehen, wenn sie Wertesysteme und Institutionen, die auf unserem Mist gewachsen sind, nicht von vornherein und ohne Vorbehalt enthusiastisch zu übernehmen bereit sind; darin liegt der legitime Aspekt der durchaus nicht hypokrisiefreien Konstruktion »asiatischer Werte« (Senghaas 1998). Sie mögen Rechte und Pflichten in anderer Weise zueinander justieren, als wir es gewöhnt sind, die wir individuellen Abwehr- und Entfaltungsrechten eindeutig die Priorität zuweisen. Sie mögen Kollektiv und Individuum in einem anderen Verhältnis sehen. Der einzelne Mensch mag ihnen nur durch seine kommunitäre Gemeinschaft Teil der Menschheit sein, also mediatisiert und nicht, wie im liberalen Universalismus, unmittelbar. Die Ergänzung der Führungsetagen durch Kooptation mag ihnen (wie der katholischen Kirche oder Unternehmen) einleuchtender und zweckmäßiger erscheinen als durch allgemeine Wahl.

Um es noch einmal deutlich zu machen: In keiner dieser Alternativen stehe ich als Person auf der anti-liberalen Seite. Ich weise nur darauf hin, dass solche Orientierungen nicht nur als herrschaftssichernde Elite-Ideologien, sondern als »Volkskultur « anderswo (noch?) ziemlich tief verankert sind. Die Prinzipien, auf denen etwa David Held (2003) seine kosmopolitische Ordnung gründen will, sind insoweit auf absehbare Zeit nicht universalisierbar. Sie sind aus unserer Kultur erwachsen und wären erst dann zur Richtschnur globaler Institutionen geeignet, wenn sie weltweit zustimmungsfähig wären. Das ist heute entschieden nicht der Fall: Dass eine Weltkultur (Meyer 2004) bereits entstanden sei, die als tragfähige Grundlage kosmopolitischer Vergemeinschaftung dienen könne, ist eine fromme Selbsttäuschung universalistischer Projektunternehmer.

\section{Kosmopolitismus als Bedrohung}

Verbinden sich diese Prinzipien, wie in der akademisch modischen Idee eines staatsauflösenden, (quasi-) demokratischen Regierens auch noch mit der Unterminierung der mühsam gewonnenen Souveränität der früher Kujonierten, wirken sie doppelt aggressiv, hypokritisch und abstoßend: Für Chinesen, ja selbst für demokratiegewöhnte Inder, muss die Zumutung, just in dem Augenblick auf ihre Staatlichkeit weitgehend zu verzichten, in dem sie durch eigenes Geschick, durch Fleiß und Beharrlichkeit die Schwelle zu einer Erweiterung ihrer nationalen Handlungsfähigkeit überschritten haben, wie ein tückischer Anschlag auf ihre Identität erscheinen: Nachdem der Westen Jahrhunderte lang in mehr oder weniger brutaler Verfolgung 
der eigenen nationalen Stärke diese Völker entmachtet und unterdrückt hat, versucht er sie erneut der mühsam wiedergewonnenen Handlungsfähigkeit zu berauben. Kosmopolitismus erscheint in dieser Perspektive wirklich als Kolonialismus mit anderen Mitteln, als westliche Herrschaftsideologie, die mit neuen Mitteln die Unterwerfung der Schwarzen, Roten, Braunen und Gelben unter die partikularen Maximen des weißen Mannes verewigen will - und das gilt selbst für eine stabile Demokratie wie die indische. Über die Wahrnehmungen in mehrheitlich moslemischen Staaten muss man nicht weiter sprechen.

Durch die Unbedingtheit des Geltungsanspruchs, den die westlichen Kosmopoliten für ihre kulturellen Werte und ihre institutionellen Lösungsvorstellungen erheben (Beispiele Held 1998; Höffe 2002; Pogge 2002; Beck 2004; Archibugi 2004), werden die ohnedies kräftigen Selbstverteidigungsinstinkte anderswo weiter gestärkt. Die Anerkennung, die den »anderen« durch die Kolonialgeschichte stets verwehrt blieb, wird weiter unter dem Vorzeichen verweigert, es könnte gar keine anderen Prinzipien des Weltregieren geben als die vom Westen ausgebrüteten. Voraussagbar wird der »Kampf um Anerkennung « in eine neue, brisante Runde gehen (Honneth 1992). Die absurde Folge tritt ein, dass der wohlmeinende Anspruch, Universalität zu befördern, stattdessen nur darauf hinausläuft, Fragmentierung zu vertiefen. Dazu tragen Machtasymmetrien und der nonchalante Umgang des kosmopolitischen Projekts mit der Gewaltproblematik maßgeblich bei.

Der Westen mag sich durch die Kämpfer der Al Qaida in seinem Innersten bedroht fühlen. Für den Rest der Welt steht er nach wie vor als ein unangreifbarer und dazu bedrohlicher Kampfstern da. Er bringt drei Viertel der Weltmilitärausgaben auf, verfügt über die bei Weitem größte Wirtschaftskraft und ist (immer noch) technisch weit überlegen. Auch in »weicher Macht« kommt ihm nichts gleich. Die Verbindung von überlegener Macht und dem Universalisierungsprogramm eigener kultureller Werte verstärkt Bedrohtheitsgefühle anderswo. Der Flurschaden, den die brutalstmögliche Version eines missionarischen Kosmopolitismus, unverkennbar versetzt mit nationalistischen Untertönen, nämlich der gegenwärtig herrschende USNeokonservatismus, anrichtet, wird uns diesbezüglich noch auf Jahre mit Aufräumarbeiten beschäftigen (Hassner 2002).

Die Kosmopoliten schreiben den Bürgern anderer Staaten Rechte zu, deren Verletzung den externen Eingriff rechtfertige (Kuper 2004; Brunkhorst 1995). Wer das »Menschenrecht auf Demokratie« propagiert (Franck 1992, 2000) und die humanitäre Intervention befürwortet, hängt das Damoklesschwert des gewaltsamen Regimewandels über sämtliche Nichtdemokratien. Denn das praktische Urteil der westlichen Zeremonienmeister des weltpolitischen Machtwalzers, zu welchem Zeitpunkt welcher nichtdemokatische Herrscher dem Verdikt des zu beseitigenden Platzhalters auf der »Achse des Bösen« verfällt, ist gänzlich unberechenbar. Der Saddam Hussein von 1985 war ein tolerierbarer autoritärer Herrscher, der von 2003 ein Tyrann, der abserviert werden musste. Der Milošević von 1995 war der begehrte Partner von Dayton, der von 1999 Ziel eines mit aller Inbrunst von Selbstgerechtigkeit vorgetragenen Luftkrieges. Ansteigende Unberechenbarkeit platziert die Beziehungen zwischen bewaffneten politischen Einheiten unentrinnbar ins Sicherheitsdi- 
lemma. Rüstungswettlauf ist seine Grammatik, ungewollte Eskalationen sind seine Satzfehler (Müller/Schörnig 2006: 40-69). Dass sich die Verunsicherten mit wirksamen Abschreckungsmitteln zu versehen suchen - Beispiele Iran und Nordkorea -, ist unter ordnungspolitischen Gesichtspunkten unschön und nicht wünschenswert, denn die dort Regierenden sind wirklich keine netten Leute. Eine kühle sicherheitspolitische Analyse wird jedoch daran nichts Verwunderliches finden: Wer droht, produziert Existenzangst, wer Existenzangst empfindet, versichert sich wirksamer Mittel der Gegenwehr.

\section{Zurück zur Bescheidenheit: Liberaler Kosmopolitismus als interkulturelles Diskursangebot}

Heißt das: Völlige Aufgabe der kosmopolitischen Idee? Nicht im Mindesten - diese Forderung ist der zentrale Denkfehler der Kommunitaristen und Poststrukturalisten (Taylor 1989; Hopgood 2000). Die empirisch identifizierbaren Anstrengungen um gemeinsame Regelungen für interdependente Handlungsfelder, die von staatlichen und nichtstaatlichen Akteuren aus allen Kulturen unternommen werden, weisen zumindest auf eine gemeinsame Problemidentifikation hin (Zürn 1998). Die Anerkennung bestehender Vielfalt schließt die Möglichkeit universell geltungsfähiger Normen und Regelungen nicht kategorisch aus. Sie schließt nur ein für allemal aus, dass einer der existierenden Partikularismen aus sich selbst heraus definieren kann, was universell gelten kann und soll. Er kann auch nicht im Vorgriff auf das Ergebnis eines noch nicht geführten Diskurses die Behauptung aufstellen, von ihm entwickelte Normen seien für alle zustimmungsfähig. Denn die Geltungskraft weltordnungsstiftender Normen kann nur aus der tatsächlichen Zustimmung aller hervorgehen, so wie das jetzt geltende Völkerrecht idealiter auch (dass ein Teil dieses Rechts Produkt einer Zeit ist, in der die westliche Staatengemeinschaft über alle anderen dominierte, mag freilich noch zu Nachkorrekturen führen).

Die Zustimmung mag erstens zustande kommen, weil es bereits jetzt tatsächlich kulturübergreifend einen geteilten Grundbestand von Gemeinsamkeit geben könnte, der als Berufungsgrundlage für solche Normen zu dienen in der Lage ist. Er mag sich zweitens durch die künftige Konvergenz heute unterschiedlicher Normensysteme über wechselseitige Interpenetration und Amalgamation ergeben. Oder auch drittens - aus langwierigen Überzeugungsprozessen in Verhandlungen, bei der die beteiligten Inseln geteilter Überzeugungen erarbeiten, wo zuvor nur trennendes Meer war (Deitelhoff 2006a, 2006b). Gelingender Universalismus ist also zwar keine Denknotwendigkeit, aber doch eine kontingente Möglichkeit, deren Realisierung nicht Frage abstrakten Räsonierens, sondern kommunikativer Praxis ist.

Es ist dabei keineswegs gewiss, aber eben auch nicht ausgeschlossen, dass der Westen tatsächlich den Schlüssel dazu in der Hand hält. Seine vermeintlich universalistischen Werte sind insofern - wie die der anderen - Diskursangebote, das heißt, der Beginn, nicht der Abschluss eines Prozesses, der zu einer tragfähigen Institutionalisierung und Verrechtlichung von weltweiten Steuerungsprozessen führen kann. 
Ein Westen, der sich auf diese Position beschränkt - Diskursinputs zu geben -, dürfte ironischerweise bessere Erfolgschancen in einem Universalisierungsprozess besitzen als mit seinen bisherigen Praktiken ungestümer Forderungen und schierer imperialer Machtpolitik.

\section{Literatur}

Archibugi, Daniele 2004: Cosmopolitan Democracy and Its Critics, in: European Journal for International Relations 10: 3, 437-475.

Archibugi, Daniele/Held, David (Hrsg.) 1995: Cosmopolitan Democracy: An Agenda for a New World Order, Cambridge.

Beck, Ulrich 2004: Der kosmopolitische Blick oder: Krieg ist Frieden, Frankfurt a. M.

Beisheim, Marianne/Dreher, Sabine/Walter, Gregor/Zangl, Bernhard/Zürn, Michael 1999: Im Zeitalter der Globalisierung? Thesen und Daten zur gesellschaftlichen und politischen Denationalisierung, Baden-Baden.

Beitz, Charles 1979: Political Theory and International Relations, Princeton, NJ.

Brunkhorst, Hauke (Hrsg.) 1999: Recht auf Menschenrechte. Menschenrechte, Demokratie und Internationale Politik, Frankfurt a. M.

Clark, Ian 1997: Globalization and Fragmentation: International Relations in the Twentieth Century, Oxford.

Deitelhoff, Nicole 2006a: Zu(m) Recht überzeugt. Die Errichtung des Internationalen Strafgerichtshofs im Spannungsverhältnis zwischen Recht und Politik, in: Becker, Michael/Zimmerling, Ruth (Hrsg.): Politik und Recht (Politische Vierteljahresschrift, Sonderheft Nr. 36), Opladen, 416-448.

Deitelhoff, Nicole 2006b: Überzeugung in der Politik. Grundzüge einer Diskurstheorie internationalen Regierens, Frankfurt a. M.

Fox, Gregory H./Roth, Brad R. (Hrsg.) 2000: Democratic Governance and International Law, Cambridge.

Franck, Thomas M. 1992: The Emerging Right to Democratic Governance, in: The American Journal of International Law 86: 1, 46-91.

Franck, Thomas M. 2000: Legitimacy and the Democratic Entitlement, in: Fox/Roth 2000, 2547.

Hassner, Pierre 2002: The United States: Empire of Force or Force of Empire? (Institute for Security Studies of the Western European Union, Chaillot Papers Nr. 54), Paris.

Held, David 2003: Cosmopolitan: Taming Globalization, in: Held, David/McGrew, Anthony (Hrsg.): The Global Transformations Reader: An Introduction to the Globalization Debate, 2. Auflage, Cambridge, 514-529.

Held, David 1998: Democracy and the Global Order: From the Modern State to Cosmopolitan Governance, Cambridge.

Höffe, Otfried 2002: Globalität statt Globalismus. Über eine subsidiäre und föderale Weltrepublik, in: Lutz-Bachmann/Bohmann 2002, 8-31.

Honneth, Axel 1992: Kampf um Anerkennung. Zur moralischen Grammatik sozialer Konflikte, Frankfurt a. M.

Hopgood, Stephen 2000: Reading the Small Print in Global Civil Society: The Inexorable Hegemony of the Liberal Self, in: Millenium: Journal of International Studies 29: 1, 125.

Krasner, Stephen D. 1999: Sovereignty: Organized Hypocrisy, Princeton, NJ.

Kuper, Andrew 2004: Democracy Beyond Borders: Justice and Representation in Global Institutions, Oxford.

Lutz-Bachmann, Matthias/Bohmann, James (Hrsg.) 2002: Weltstaat oder Staatenwelt? Für und wider die Idee einer Weltrepublik, Frankfurt a. M. 
Harald Müller: Liberaler Kosmopolitismus: Eine partikularistische Emanation mit Unverträglichkeiten und Nebenwirkungen

Meyer, John W. 2004: Weltkultur. Wie die westlichen Prinzipien die Welt durchdringen, Frankfurt a. M.

Müller, Harald 1998: Das Zusammenleben der Kulturen, Frankfurt a. M.

Müller, Harald/Schörnig, Niklas 2006: Rüstungsdynamik und Rüstungskontrolle. Eine exemplarische Einführung in die Internationalen Beziehungen, Baden-Baden.

Pogge, Thomas 2002: Kosmopolitanismus und Souveränität, in: Lutz-Bachmann/Bohmann 2002, 125-171.

Rupnik, Jacques 2006: Die Dilemmata der Europäischen Union, Transit: Europäische Revue 30, 5-19.

Senghaas, Dieter 1998: Zivilisierung wider Willen, Frankfurt a. M.

Taylor, Charles 1989: Sources of the Self, Cambridge, MA.

Zürn, Michael 1998: Regieren jenseits des Nationalstaats. Globalisierung und Denationalisierung als Chance, Frankfurt a. M.

Zürn, Michael 2001: Politische Fragmentierung als Folge der gesellschaftlichen Denationalisierung?, in: Loch, Dietmar/Heitmeyer, Wilhelm (Hrsg.): Schattenseiten der Globalisierung. Rechtsradikalismus, Rechtspopulismus und separatistischer Regionalismus in westlichen Demokratien, Frankfurt a. M., 111-139. 